\section{Radiologin für herausragende wissenschaftliche Arbeiten mit dem Gustav-Bucky-Preis 2013 ausgezeichnet}

\author{
Dr. med. Diane Renz von der Charité erhält Preis der Berliner \\ Röntgen-Gesellschaft Röntgenvereinigung zu Berlin und \\ Brandenburg e. V. (BRG-RBB)
}

Berlin, 9. April 2013. Bereits zum zweiten Mal würdigt die Berliner RöntgenGesellschaft einen Wissenschaftler mit dem Gustav-Bucky-Preis. Die diesjährige Preisträgerin ist Frau Dr. med. Diane Renz von der Charité Berlin.

Dr. Diane Renz erhielten am 9. April 2013 den Gustav-Bucky-Preis für ihre in der Zeitschrift Investigative Radiology publizierte wissenschaftliche Arbeit „Pharmacokinetic Approach for Dynamic Breast MRI to Indicate Signal Intensity Time Curves of Benign and Malignant Lesions by Using the Tumor Flow Residence Time", in der sie die pathophysiologischen Vorstellungen der Pharmakokinetik von Mammaläsionen untersucht. Dr. med. Beate Rehbock, Vorstandsmitglied der Berliner Röntgen-Gesellschaft, sagt in ihrer Begründung zur Preisverleihung: „Frau Dr. Renz kann auf beachtliche wissenschaftliche Publikationen als Erstautorin in hochdotierten nationalen und internationalen Zeitschriften verweisen. Die Themen der Arbeiten einschließlich des Posterpreises und ihr Curriculum belegen eine vorangegangene intensive wissenschaftliche Auseinandersetzung mit dem Schwerpunkt Mammadiagnostik und legitimieren die Bewerberin zusätzlich für die Prämierung der eingereichten Publikation.“

Dr. Renz, 1973 in Sindelfingen geboren, schloss 2000 ihr Medizinstudium an der RHTW Aachen ab. Ihre Approbation als Ärztin erhielt sie 2003. Seit Januar 2010 ist sie Fachärztin für Radiologie. Sie ist außerdem Diplom-Journalistin (Abschluss 2005, LMU München) und arbeitet als freie Mitarbeiterin für das Ressort Wissenschaft der Frankfurter Allgemeinen Sonntagszeitung.

Mit dem Gustav-Bucky-Preis wird der wissenschaftliche Nachwuchs in der Radiologie gefördert. Der Preis dient auch dem Gedenken an den Namensgeber Gustav Bucky, der mit seinem Schaffen einen wesentlichen Beitrag zum Fortschritt der Radiologie geleistet hat.

Gustav Bucky wurde am 3. September 1880 in Leipzig geboren. Er publizierte über 100 wissenschaftliche Arbeiten und besaß 94 deutsche und amerikanische Patente auf dem Gebiet der Röntgentechnik. Bekannt wurde er vor allem durch die Erfindung der nach ihm benannten Bucky-Blende, einem wichtigen Beitrag zur Reduktion der Streustrahlung und Qualitätsverbesserung in der Röntgendiagnostik. 1933 emigrierte Bucky von Berlin nach New York. Dort war er unter anderem Hochschullehrer am New York College of Medicine sowie am Albert Einstein College of Medicine. Gustav Bucky starb 1963 in New York. Die Röntgenvereinigung zu Berlin und Brandenburg ehrt mit diesem Preis Gustav Bucky sowie seine radiologischen Kollegen, die zwischen 1933 und 1945 aus ihrer Tätigkeit verbannt oder zur Emigration gezwungen wurden.

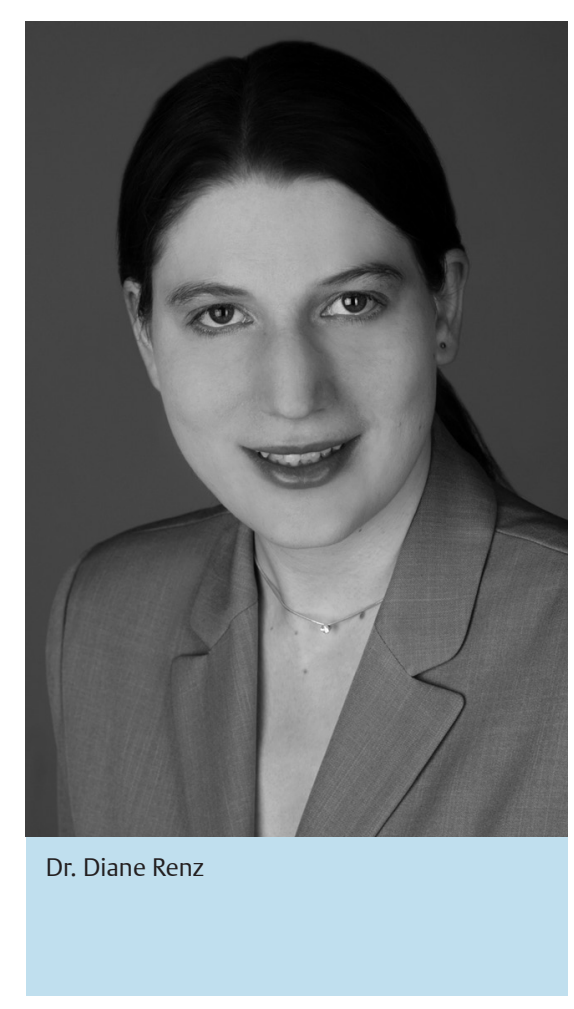

Die am 18.März 1898 gegründete Röntgen-Vereinigung zu Berlin ist die älteste radiologische Fachgesellschaft. Sie organisierte 1905 den Röntgenkongress in Berlin, auf dem die Deutsche Röntgengesellschaft e.V. gegründet wurde. Nach der Wiedervereinigung und dem Zuwachs aus dem Ostteil Berlins und aus Brandenburg wurde 1991 der Name umgeändert in „Berliner Röntgengesellschaft - Röntgenvereinigung zu Berlin und Brandenburg“. Heute zählt die BRG-RBB mehr als 400 Mitglieder.

Pressekontakt:

\section{Florian Schneider}

Presseverantwortlicher

Deutsche Röntgengesellschaft e.V.

Tel.: 030-916 07019

Fax: 030-916 07022

E-Mail: schneider@drg.de

Internet: www.drg.de 